Cinémas

Revue d'études cinématographiques

Journal of Film Studies

Sophie-Jan Arrien et Jean-Pierre Sirois-Trahan (dir.), Le

montage des identités, Québec, Presses de l'Université Laval, 2008, $170 \mathrm{p}$.

\title{
Gwenn Scheppler
}

Volume 21, numéro 1, automne 2010

URI : https://id.erudit.org/iderudit/1005636ar

DOI : https://doi.org/10.7202/1005636ar

Aller au sommaire du numéro

Éditeur(s)

Cinémas

ISSN

1181-6945 (imprimé)

1705-6500 (numérique)

Découvrir la revue

Citer ce compte rendu

Scheppler, G. (2010). Compte rendu de [Sophie-Jan Arrien et Jean-Pierre

Sirois-Trahan (dir.), Le montage des identités, Québec, Presses de l'Université

Laval, 2008, 170 p.] Cinémas, 21(1), 205-214. https://doi.org/10.7202/1005636ar d'utilisation que vous pouvez consulter en ligne.

https://apropos.erudit.org/fr/usagers/politique-dutilisation/ 
Sophie-Jan Arrien et Jean-Pierre Sirois-Trahan (dir.), Le montage des identités, Québec, Presses de l'Université Laval, 2008, $170 \mathrm{p}$.

L'ouvrage collectif dirigé par Sophie-Jan Arrien et Jean-Pierre Sirois-Trahan est paru depuis quelque temps déjà. Mais ses propositions méritent un examen plus attentif que celui qui lui a été jusqu'à présent consacré. La première raison en est l'actualité (indémodable) de son sujet, soit la constitution des identités individuelles et collectives à l'ère postmoderne. Mais cela seul ne suffirait pas à justifier son intérêt n'était l'originalité de l'approche proposée: analyser, selon une perspective interdisciplinaire, la (dé)construction de l'identité à l'aune du concept de montage, emprunté au cinéma.

Le livre part d'un constat: alors que la notion d'identité semblait avoir été bannie par les concepts postmodernes de diffusion et de déconstruction, elle continue «de s'imposer comme un problème lancinant avec lequel nous ne finissons pas d'en découdre» (p. 2). Cette identité qui ne veut pas passer, ou qui ne cesse de s'imposer, est désormais "virtuelle", "fabriquée", "flottante», "fragmentée» et "clivée»; façonnée par les aléas des possibles et de l'expérience, elle ne marque plus une existence stable mais se rebâtit continuellement autour du principe de devenir-autre.

Cette nouvelle identité versatile et fluide est l'objet de tous les soins de nombreuses disciplines des sciences humaines, qui ont toutefois la fâcheuse habitude de travailler isolément. Lidentité est devenue un concept multidisciplinaire dont l'analyse et la compréhension changent - parfois totalement - d'une discipline à l'autre. Une approche interdisciplinaire serait donc devenue nécessaire, d'où l'idée même de ce livre où Jean-Pierre Sirois-Trahan et Sophie-Jan Arrien proposent de croiser les 
approches de la philosophie, des études cinématographiques et de l'histoire de l'art.

Dans Le montage des identités, les auteurs ont donc envisagé d'analyser et de modéliser les conceptions actuelles de l'identité à partir «de la valeur heuristique du concept cinématographique de montage» (p. 5). Ou, pour le dire autrement, ils ont essayé de voir dans quelle mesure l'importation du concept de montage dans d'autres champs des sciences humaines ouvrait des perspectives neuves pour comprendre les mutations postmodernes du concept d'identité. Celle-ci fonctionne selon les auteurs sur l'emprunt, le collage, la fragmentation et l'éclatement pour former un tout ouvert. Elle ressemble à la fois au montage d'un film et à l'expérience "par procuration" de son sujet-spectateur. Par la démarche de ce livre, ce serait «l'expérience artistique qui viendrait à la rescousse de l'expertise philosophique et réciproquement» (p. 4).

Cet ouvrage, qui tire son origine d'un colloque (Le montage des identités, 17 mai 2006, Université McGill, Association francophone pour le savoir — Acfas), propose une approche originale et audacieuse, surtout quand on connaît la disparité constitutive des colloques interdisciplinaires. Comment les auteurs proposent-ils d'arriver à leurs fins? Le texte se scinde radicalement en deux grandes parties. La première traite du concept polysémique d'identité selon une perspective théorique où le montage cinématographique, dans toute sa complexité et sa richesse, sert de fil directeur à la réflexion. La seconde suit plutôt le cheminement inverse: partir de cas d'œuvres artistiques «montées» afin d'en extraire les constructions identitaires particulières. Dans ce compte rendu, nous avons choisi de nous concentrer sur la valeur propre de chaque contribution plutôt que sur le plan d'ensemble, ce qui ne veut pas dire que nous n'en parlerons pas.

Le premier texte, "Amour libre et identité morale, à propos de Peter Sloterdijk et de quelques autres" (Marc Hunyadi, professeur de philosophie à l'Université de Louvain-la-Neuve), revient sur l'intérêt et l'effectivité du concept de montage dans l'approche de l'identité. Il épouse une perspective épistémologique dans laquelle l'auteur met en évidence l'actualité du 
concept de montage dans des domaines aussi divers que l'esthétique de l'œuvre d'art, les mathématiques ou le génie génétique. Mais son objet central est le déploiement d'une réflexion sur l'identité dans l'œuvre philosophique de Sloterdijk à travers les trois états de l'identité du "sujet analytique» contemporain (conservation, expérimentation et intensification de soi). Hunyadi expose comment ce sujet contemporain, issu d'un processus de déshéritement identitaire, se retrouve face au Carré noir sur fond blanc de Malevitch, "un écran interne vide» (p. 21). Il aurait alors toute liberté d'expérimenter et de se créer, par «l'invention poétique de soi» (p. 23).

Ce texte est foisonnant, dans la présentation des concepts comme dans celle des références et des auteurs, au point de nuire parfois à la lisibilité. Il n'en constitue pas moins une mise au point intéressante sur les multiples conceptions du soi qui se rencontrent et s'affrontent dans les sociétés postmodernes. On peut regretter en revanche la conclusion un peu banale et prescriptive d'Hunyadi :

Ce serait une erreur de penser que l'identité postmoderne, quoique l'on désigne exactement par là, remplace les identités traditionnelles; car elle s'y ajoute. La logique ne doit pas être exclusive mais inclusive: le et doit remplacer le ou (p. 30).

Une telle affirmation, bien que louable et défendable, semble à la fois relever du lieu commun et du discours politique.

Le texte d'Arrien (docteure en philosophie de Paris IV et professeur à l'Université Laval), "Montage narratif et constitution performative du soi (Paul Ricoeur, Wilhelm Schapp et Antonin Artaud) ", élabore une réflexion autour d'une réplique d'Artaud, "La vie est un coup monté ${ }^{1}$ ", afin de voir quelles réponses Schapp et Ricoeur lui ont apportées.

Pour procéder, Arrien explique comment la philosophie a pu, grâce à Nietzsche, remettre en cause l'identité à soi du sujet cartésien ("mêmeté»), en mettant en évidence sa dimension proprement langagière (c'est-à-dire externe à soi). Cette étape permit ensuite à Ricœur de proposer une identité narrative reposant sur la dialectique entre idem et ipse, une identité narrative qui n'est donc jamais "ni parfaitement stable ni définitive» 
(p. 34), où la personne devient "un personnage intriqué dans ses propres expériences vécues et luttant narrativement contre l'éparpillement de soi » (p. 35). Mais là où Ricœur conçoit encore un sujet élaborant le récit de son identité à travers celui de ses expériences, Schapp l'imagine plus radicalement «empêtré dans une histoire collective [...] dont les empêtrements et intrications multiples sont intégralement constitutifs de son identité» (p. 38) : l'identité narrative de l'individu lui échappe donc en grande partie dans des récits collectifs qu'il ne contrôle ni ne perçoit clairement. Selon cette conception, identité individuelle et collective seraient intimement liées. Enfin, Arrien revient à Artaud et à sa réplique inaugurale, dont le sens premier serait la conclusion de cette dépossession progressive du sujet depuis Nietzsche: "c'est le langage qui tient l'être en son pouvoir et non l'inverse» (Artaud 1994, p. 148), un langage envoûtant le sujet. Ce deuxième texte est donc à la fois érudit et éclairant. Il est intéressant à plus d'un titre, notamment en raison de sa mise en avant d'une évolution du concept d'identité dans la philosophie. La problématisation de l'identité comme narration à travers l'analyse des discours d'Artaud, Ricœur et Schapp est originale et propose une alternative aux sempiternelles approches un peu binaires du sujet «déconstruit» et "virtuel» de l'ère postmoderne.

Le texte de Pierre-Antoine Chardel (docteur en philosophie et sciences sociales de l'EHESS de Paris et maître de conférences à l'Institut National des Communications) nous semble plus problématique que les précédents. Il s'intitule "L'identité de la conscience à l'épreuve des industries culturelles ", et en substance, il s'agit d'un commentaire exégétique de la pensée de Bernard Stiegler sur le cinéma dans La technique et le temps.

Pour Stiegler la conscience fonctionne comme le cinéma, ce qui permet à ce dernier d'avoir une prise directe sur elle. Son emprise serait d'autant plus puissante que le film est un «objet temporel ", un flux qui vient syntoniser le cerveau du spectateur (réduit au statut d'un vulgaire processeur contrôlé à distance en utilisant la bonne fréquence). Dans une perspective heideggérienne, le cinéma serait, avec la télévision, une émanation des industries de programme (ou culturelles) qui puisent dans la 
conscience et l'esprit des individus et opèrent "une synchronisation des diachronies constitutives des cultures, c'est-à-dire aussi des consciences" (p. 58): derrière une formulation alambiquée, le cinéma est une nouvelle fois accusé d'être une entreprise d'uniformisation "mortifere» des masses. Pour Stiegler, ce qui au cinéma est perdu, c'est l'altérité radicale de la conscience par rapport au texte écrit, que décrie Platon et que salue Nietzsche pour la liberté d'interprétation qu'elle offre. Il est un peu regrettable que cette exégèse attende trop longtemps pour remettre en question les postulats de Stiegler et leur consacre trop peu de place (quelques lignes en conclusion): Stiegler ne néglige-t-il pas le caractère hybride du cinéma (qui est aussi produit d'écriture) ? Le spectateur est-il aussi passif qu'il le croit? Les processus de réception sont-ils aussi uniformisés qu'il se plait à le croire?

Le dernier texte de cette première partie, écrit par Manola Antonioli (docteure en philosophie et sciences sociales de l'EHESS de Paris, affiliée au Collège international de philosophie de Paris), est lui aussi une exégèse: "Félix Guattari: l'identité individuelle et collective comme hétérogénèse ». Ce texte a l'indéniable mérite de redonner sa place au deuxième terme de l'agencement collectif d'énonciation de Capitalisme et schizophrénie et de quelques autres classiques deleuziens. Antonioli se concentre en particulier sur le principe de transversalité, le lien par lequel des termes hétérogènes en devenir viennent à l'existence par leur rencontre même (on ne sort donc pas des catégories deleuziennes). Dans cette perspective, l'identité collective ou individuelle est plurielle, polyphonique, elle est induite par un processus d'hétérogenèse au cœur duquel se trouve le langage (où l'on retrouve, en moins dramatique, l'idée d'une identité narrative développée par Arrien). L'auteur, par une transition étrange, passe ensuite à des considérations philosophico-sociologiques guattariennes sur le rapport territoire/identité, où elle réitère le postulat qui veut que, dans «le contexte de haute déterritorialisation des sociétés industrielles contemporaines", le mélange subjectif «d'attachement archaïsant aux traditions culturelles et à tous les enracinements identitaires» n'a plus vraiment sa place (p. 83). Elle conclut par une exhortation à réinventer la vie quotidienne et 
l'espace démocratique. Ce texte néglige le montage, le thème même du collectif. S’il éclaire un peu le lecteur sur des aspects méconnus de la pensée de Guattari, il n’apporte pas grand-chose de nouveau par rapport aux trois textes précédents.

La deuxième partie de l'ouvrage part des œuvres afin d'étudier quelles constructions identitaires elles mettent en place.

"Le Devenir-Québécois chez Pierre Perrault», rédigé par Jean-Pierre Sirois-Trahan (docteur de l'Université de Montréal et de l'Université Paris III, professeur adjoint au Département des littératures de l'Université Laval), revisite et prolonge les réflexions développées par Gilles Deleuze autour du cinéma de Perrault dans Limage-temps, chapitre six. L'idée générale est que le cinéma de Perrault a déstabilisé l'identité collective des Québécois lors de la Révolution tranquille en poussant à sa limite la capacité du cinéma à produire de la pensée (le film comme flux mental). Perrault serait parvenu à questionner en profondeur les processus identitaires collectifs du Québec, en mettant en place des "agencements collectifs d'énonciation": le discours n'appartient pas au cinéaste ou à un personnage en particulier, ni à une instance abstraite; il est un tressage de paroles et de voix, d'expériences subjectives, où tout à la fois les personnages sintercèdent le cinéaste pour prendre la parole, et où le cinéaste sintercède les personnages pour élaborer son propre discours ${ }^{2}$. La prise de parole collective est l'objet même des films de Perrault, et le spectateur est incité par la ligne du montage à participer à son tour, à ajouter sa voix au concert discordant.

Le texte de Sirois-Trahan vaut moins par l'analyse qu'il fait du cinéma de Perrault (par ailleurs fort juste) que par sa mise en perspective de la signification de l'œuvre de Perrault dans la pensée profonde du philosophe. Ce texte propose des remarques fertiles sur la façon dont le concept "d'intercesseur" a ressurgi dans les travaux de Deleuze et Guattari sur les "personnages conceptuels", ces "instances énonciatrices à la troisième personne qui pensent à travers les philosophes et créent les concepts; c'est l'autre en moi» (p. 102). Cette mise en abyme 
ouvre des perspectives fascinantes sur les transferts sinueux de la pensée et de l'expérience, menant de Grand-Louis à Gilles Deleuze.

"L'épreuve identitaire dans les carnets de voyage filmés ", écrit par Lise Gantheret (documentariste et docteure en recherches audiovisuelles et cinématographiques de Paris III), propose une réflexion très intéressante sur la recherche d'altérité et l'exotisme. Elle compare deux formes de rencontre filmique apparemment similaires mais qui s'avèrent en fin de compte profondément incompatibles: les documentaires télévisuels "Lonely Planet» (1998-2010) et L'Inde fantôme (1968) de Louis Malle.

C'est la posture de l'intercesseur qui intéresse Gantheret. D’un côté, Malle fait du cinéma direct, ce qui implique une posture de "cinéaste biographe » dont l'expérience vécue, intime et subjective, est au cœur du film: la rencontre de l'Autre est aussi une mise en danger de soi, une implication. Les documentaires "Lonely Planet", en revanche, répondent plus à des normes institutionnelles qui doivent être réitérées de film en film, montrant l'Autre comme un indigène sympathique et original dont, au final, on ne sait pas grand-chose. Le cinéaste biographe s'implique en reconnaissant à la fois l'altérité radicale de l'Autre et en se focalisant sur ce qui ne fait pas consensus: la marge, la folie et la pauvreté. La démonstration de Lise Gantheret est intéressante mais on se questionne tout de même sur le bienfondé des comparaisons. Elle oppose les cinéastes biographes aux documentaristes de "Lonely Planet» avant tout dans leur démarche et leur posture, les premiers semblant avoir des exigences éthiques supérieures aux seconds, ce qui leur permettrait une rencontre plus authentique. Or, d'une part, l'histoire du cinéma direct est jonchée de rencontres ratées et de malentendus. D'autre part, la posture "éthique» du cinéaste biographe qu'est Louis Malle pourrait elle-même être critiquée, quand Lise Gantheret explique qu'il

prend pour exemple la société Todas aux mœurs sexuelles particulièrement libres[,] qu'il considère comme modèle idéal parce que non hypocrite. Les jeunes filles sont très précocement initiées à l'amour par un homme du village. Le sexe, loin d'être tabou, s'affiche comme n'importe quelle autre pratique (p.119). 
En revanche, les documentaires «Lonely Planet» pourraient être remis en question sur le plan éthique justement parce que, entre autres, la dimension sexuelle des cultures rencontrées n'y est pas abordée. La dichotomie entre "Lonely Planet» et cinéma biographique aurait donc mérité quelques nuances.

Hudson Moura (documentariste et docteur en littérature et cinéma de l'Université de Montréal) explore ensuite les trajets de deux cinéastes canadiens d'origine arménienne (Atom Egoyan et Gariné Torossian), à la lumière des concepts d'intermédialité et d'interculturalité tels qu'ils se développent dans leurs films respectifs: Calendar (Atom Egoyan, 1993) (long métrage de fiction) et Girl from Moush (Gariné Torossian, 1993) (court métrage expérimental). Les films sont d'autant mieux choisis qu'ils portent précisément sur un questionnement intérieur, une exploration des questions d'identité culturelle chez des sujets de la diaspora arménienne qui tentent de renouer des liens avec leur histoire, de retrouver les traces d'un passé qui les hante sans même qu'ils l'aient vécu. Le mérite de ce texte tient particulièrement dans le fait de questionner l'influence du cinéma en tant que média mémoriel sur la dialectique qui relie la constitution des identités individuelle et collective.

Le dernier texte, rédigé par François Le Tourneux (artiste et doctorant en histoire de l'art à l'Université de Montréal), s'intitule "Hétérogénéité stylistique et collage», et traite donc principalement d'art pictural selon une perspective très novatrice. Ce texte, qui ressemble quelque peu par sa rigueur et sa structure à une introduction de thèse, boucle le parcours à la fois conceptuel et interdisciplinaire de l'ouvrage.

Hétérogénéité et collage sont, selon l'auteur, les deux formes de montage caractéristiques de l'évolution des pratiques picturales modernes. Paradoxalement pourtant, il prend soin de nous expliquer qu'une infime minorité d'artistes (dont Picasso) ont adopté l'hétérogénéité stylistique, et que l'histoire de l'art n'a quasiment pas retenu ou envisagé ce concept, ou alors l'a sévèrement condamné comme s'il s'agissait d'un style opportuniste (ainsi de Krauss à propos de Picasso). Le collage serait une forme d'hétérogénéité interne propre à l'œuvre individuelle et visuellement perceptible, tandis que l'hétérogénéité stylistique serait 
dépendante d'une connaissance importante du corpus global dans lequel l'œuvre individuelle s'inscrit: alors que le montage est visuellement perceptible et compréhensible dans le collage, il est en revanche implicite et renvoie à une connaissance extérieure à l'œuvre individuelle dans le cas de l'hétérogénéité stylistique. Le concept de montage serait donc un paradigme important de l'art pictural moderne, de sa compréhension et de sa perception, entraînant un rapport dialogique entre les œuvres, mais aussi entre l'œuvre et celui qui la regarde. Ce dernier texte, qui parvient avec brio à illustrer la relation profonde de l'art pictural avec le montage cinématographique, achève de prouver l'intérêt global de la démarche adoptée par les deux auteurs de cet ouvrage collectif.

Il est difficile a priori de percevoir un lien clair entre tous ces textes, dont les objets sont souvent disparates et les domaines de référence habituellement hermétiques les uns aux autres. On remarque néanmoins une certaine prédominance, ou surprésence de la philosophie deleuzienne, qui constitue plus un fil directeur qu'un leitmotiv, mais on se souviendra que Deleuze fut parmi les premiers à oser faire du saut interdisciplinaire une attitude souhaitable de la connaissance et de l'analyse des phénomènes humains modernes. Deleuze, dans Qu'est-ce que la philosophie?, compare la pensée à un monologue intérieur, qui accède à "une puissance immanente de création une fois libérée du vrai en tant que paradigme supposé» (Deleuze et Guattari 1991, p. 133). Ce qui est vrai pour la fabulation des personnages de Perrault l'est aussi de la pensée créatrice: il est parfois nécessaire de s'extraire des systèmes institutionnalisés de connaissance et d'analyse pour trouver du nouveau.

C'est un peu ce qu'ont fait les auteurs ayant collaboré à ce livre: ils ont osé sortir de leur champ de compétences pour dialoguer et proposer des hypothèses inusitées venues de la rencontre elle-même. Cela peut certes paraître discutable dans certains textes pris individuellement, et le concept d'identité est parfois un peu relégué au second plan. Mais, justement, la force des textes réside plus encore dans leur mise en relation que dans leurs indéniables qualités intrinsèques, dans cette espèce de cheminement auquel est convié le lecteur à travers une lecture 
(un parcours) hétéroclite et interdisciplinaire. Et, alors, l'intérêt du concept de "montage des identités " apparaît pleinement, en filigrane; sa logique comme sa structure traversent les textes, émergeant de l'assemblage des réflexions disparates. Car le livre fonctionne lui-même sur le principe du montage, l'interstice entre les textes étant le lieu final de la lecture et de la réflexion, une lecture évidemment dialogique. Pari réussi, donc.

Gwenn Scheppler

Université Laval

\section{NOTES}

1. Cahiers préparatoires à la conférence du Vieux-Colombier du 13 janvier 1947 (Artaud 1994, p. 61).

2. Voir à ce propos la réflexion de Gilles Deleuze sur le cinéma de Pierre Perrault dans Limage-temps (1985, p. 195-197).

\section{RÉFÉRENCES BIBLIOGRAPHIQUES}

Artaud 1994: Antonin Artaud, Euvres complètes, tome XXVI: Histoire vécue d'Artaud-Mômo. Tête-à-tête, Paris, Gallimard, 1994.

Deleuze 1985: Gilles Deleuze, Limage-temps, Paris, Minuit, 1985.

Deleuze et Guattari 1991: Gilles Deleuze et Félix Guattari, Qu'est-ce que la philosophie?, Paris, Minuit, 1991. 